(2) Open Access Full Text Article

\title{
Agonism of Gpr40 Protects the Capacities of Epidermal Stem Cells (ESCs) Against Ultraviolet-B (UV-B)
}

This article was published in the following Dove Press journal: Drug Design, Development and Therapy

\author{
Chengkuan Sun ${ }^{1} *$ \\ Yulin $\mathrm{Li}^{2, *}$ \\ Xianglan $\mathrm{Li}^{3}$ \\ Jing Sun $^{3}$
}

'Department of Hand Surgery, The Third Hospital of Jilin University, Changchun, Jilin I30033, People's Republic of China; ${ }^{2}$ Key Laboratory of Pathobiology, Ministry of Education, Jilin University, Changchun, Jilin I30033, People's Republic of China; ${ }^{3}$ Departmentof Dermatology, The Third Hospital of Jilin University, Changchun, Jilin 130033.

People's Republic of China

*These authors contributed equally to this work
Correspondence: Jing Sun

$\mathrm{Tel} / \mathrm{Fax}+86-043$ I-84995996

Email sunjing99@jlu.edu.cn
Introduction: Skin damage due to overexposure to ultraviolet B (UV-B) radiation can lead to the development of cancers and reduce the skin's functionality as a vital protective barrier. Epidermal stem cells (ESCs) are pluripotent cells responsible for skin regeneration and healing. Upon exposure to UV-B radiation, ESCs produce excess amounts of reactive oxygen species (ROS) and inflammatory cytokines. However, the functional protection of ESCs is not fully explored. G-protein coupled G protein-coupled receptor 40 (Gpr40) is a free fatty acid receptor that is emerging as a potential treatment target for various diseases. Gpr40 has been found to be expressed in various cell types.

Methods: ESCs were exposed to UV-B at the intensities of 25,50 , and $100 \mathrm{~mJ} / \mathrm{cm}^{2}$ for $24 \mathrm{~h}$ using TL $20 \mathrm{~W} / 12$ RS UV lamps. ESCs were treated with UV-B at $50 \mathrm{~mJ} / \mathrm{cm}^{2}$ in the presence or absence of 25 or $50 \mu \mathrm{M}$ of the Gpr40 agonist GW9508 for $24 \mathrm{~h}$. The gene expression of the Wnt1 pathway and proinflammatory cytokines were evaluated. To antagonize Gpr40 expression, ESCs were treated with $10 \mu \mathrm{M}$ GW1100.

Results: Our findings demonstrate that Gpr40 agonism can reduce the production of ROS as well as the expression of interleukins $1 \beta$ and 8 , two key proinflammatory cytokines. We demonstrate that agonism of Gpr40 can rescue the reduction in integrin $\beta 1$ and Krt19 induced by UV-B exposure, thereby improving the capacities of ESCs to resist UV-B damage. Moreover, we show that the effects of Gpr40 agonism observed in our experiments are mediated through the Wnt/ $\beta$-catenin canonical signaling pathway, as evidenced by the expression of Wnt1 and cyclin D1.

Conclusion: Our findings present evidence of the role of Gpr40 agonism in mediating the protective capacities of ESCs against insult from UV-B radiation.

Keywords: Gpr40, G protein-coupled receptors, ultraviolet-B radiation, epidermal stem cells, Wnt, GW9508

\section{Introduction}

The skin is the largest and one of the most important organs of the human body. It serves as the first line of defense against environmental toxins and pathogens. ${ }^{1}$ Epidermal stem cells (ESCs) are key mediators of skin homeostasis. These rare cells are responsible for many of the protective effects and regular functions of the epidermis, including skin development and repair. ${ }^{2}$ However, these processes can be disrupted under certain conditions. Ultraviolet-B (UV-B) is naturally emitted from the sun and largely absorbed by the ozone, but some UV-B still makes it to the Earth's surface. Overexposure of the skin to UV-B radiation leads to inhibition of 
the protective functions of ESCs, and prolonged exposure can lead to numerous types of cancer. ${ }^{3} \mathrm{UV}-\mathrm{B}$ has also been shown to significantly increase the inflammatory response and disrupt the oxidant/antioxidant balance. Proinflammatory cytokines such as interleukin-1 $\beta$ (IL-1 $\beta$ ) and interleukin-8 (IL-8) are significantly upregulated upon exposure to UV-B radiation. ${ }^{4,5}$ Additionally, UV-B induces a state of oxidative stress by triggering an increase in the production of reactive oxygen species (ROS). ROS are important biphasic free radicals that can damage the function of unfolded proteins. ${ }^{6} \mathrm{UV}-\mathrm{B}$ also damages the functions of other essential contributors to skin homeostasis, such as the Wnt signaling pathway, integrin $\beta 1$, and keratin 19 (Krt19). The Wnt pathway plays an essential role in cell signaling as well as skin tissue regeneration. ${ }^{7}$ Integrin $\beta 1$ is a cell surface receptor that binds with other members of its family to form integrin complexes. These complexes act as collagen receptors. Integrin $\beta 1$ is involved in tissue regeneration and the regulation of skin homeostasis, the immune response, and cell migration. ${ }^{8}$ Keratin type I cytoskeleton 19 (Krt19) is an intermediate filament protein of $40 \mathrm{kDa}$ that plays a key role in the maintenance of epithelial cells. ${ }^{9}$ The Wnt1/ $\beta$-catenin canonical signaling pathway has been demonstrated to mediate radiation resistance in mouse mammary progenitor cells as well as the protective effect of TGR5 against UV radiation. ${ }^{10,11}$ Thus, increasing the activation of the $\mathrm{Wnt} 1 / \beta$-catenin pathway may serve as a potential treatment approach.

$\mathrm{G}$ protein-coupled receptor 40 (Gpr40), also known as free fatty acid receptor 1 (FFAR1), is a member of the free fatty acid receptor family. ${ }^{12}$ This receptor is involved in the regulation of a number of bodily functions, including but not limited to the enteroendocrine system and the maintenance of healthy weight. Gpr40 is mainly recognized for its involvement in free fatty acidmediated signaling and incretin secretion. ${ }^{13,14}$ As Gpr40 is involved in diverse tissues and cellular processes, so too is it involved in the pathogenesis of various diseases. These include kidney fibrosis and nonalcoholic fatty liver disease. ${ }^{15,16}$ Furthermore, Gpr40 agonism has demonstrated protective qualities in Alzheimer's disease. ${ }^{17}$ The role of Gpr40 in the context of epidermal cells and UV-B damage is not yet understood. GW9508 is a Gpr40 agonist that has been recently used in studies on atherosclerosis and insulin secretion. ${ }^{18,19}$ In the present study, we demonstrate the ability of Gpr40 agonism by GW9508 to rescue the protective functions of ESCs exposed to UV-B radiation. These findings demonstrate a novel use of GW9508 as a treatment for UV-Binduced skin damage.

\section{Materials and Methods Cell Culture and Treatment}

ESCs were isolated from eight-week-old C57BL/6 female mice using skin samples taken from the backs of the mice, as previously described. ${ }^{20}$ All animal experiments were approved by the Institutional Animal Care and Use Committee at the Third Hospital of Jilin University. Studies were performed in accordance with the protocol of the Institutional Animal Care and Use Committee at the Third Hospital of Jilin University. Briefly, the isolated ESCs were maintained in Dulbecco's Modified Eagle's Medium (DMEM)/F12 medium supplemented with $10 \%$ fetal bovine serum (FBS), 1\% glutamine, 1\% antibiotics (penicillin/streptomycin), and $4 \mathrm{ng} / \mathrm{mL}$ fibroblast growth factor-basic (bFGF). Murine N43/5 cell line was used as a positive control in the Gpr40 detection experiment. ${ }^{21}$ To determine the effects of UV-B radiation on the expression of Gpr40, ESCs were exposed to UV-B at the intensities of 25,50 , and $100 \mathrm{~mJ} / \mathrm{cm}^{2}$ (wavelength 290-320 nm, maximum peak $311 \mathrm{~nm}$ ) in Dulbecco's Modified Eagle's Medium (DMEM)/F12 medium with 10\% fetal bovine serum (FBS) for $24 \mathrm{~h}$ using TL $20 \mathrm{~W} / 12 \mathrm{RS}$ UV lamps. ESCs were treated with UV-B at $50 \mathrm{~mJ} / \mathrm{cm}^{2}$ in the presence or absence of 25 or $50 \mu \mathrm{M}$ GW9508 for 24 h. For the Gpr40 antagonism experiment, cells were treated with $10 \mu \mathrm{M} \mathrm{GW} 1100$.

\section{Real-Time Polymerase Chain Reaction (PCR)}

To determine the mRNA expression of the target genes, total RNA was extracted from ESCs using Trizol reagent (Invitrogen, USA). A First Strand cDNA Synthesis Kit (Thermo Fisher Scientific, USA) with reverse transcriptase (RT) enzymes and $1 \mu \mathrm{g}$ RNA was used to synthesize the complementary DNA (cDNA). Then, $20 \mu \mathrm{g}$ cDNA was used for quantitative real-time PCR analysis with SYBR Green MasterMix (Roche, Switzerland) on an ABI 7500 Real-Time PCR platform. The expression of the target genes was calculated using the $2^{-\Delta \Delta \mathrm{Ct}}$ method and normalized to glyceraldehyde-3-phosphate dehydrogenase (GAPDH). The primer sequences are listed in Table 1. 
Table I The Prime Rs Sequences

\begin{tabular}{|c|c|c|}
\hline Target Gene & Upstream Sequence (5'-3') & Downstream Sequence (5'-3') \\
\hline Gpr40 & TGGCCCACTTCTTCCCACTC & CAGGAGAGAGAGGCTGAAGC \\
\hline IL-I $\beta$ & TGGGCCTCAAAGGAAAGAAT & CAGGCTTGTGCTCTGCTTGT \\
\hline IL-8 & ACTTCCAAGCTGGCCGTGGCTCTCTTGGCA & TGAATTCTCAGCCСTCTTCAAAAACTTCTC \\
\hline integrin $\beta I$ & ACACCGACCCGAGACCCT & CAGGAAACCAGTTGCAAATTC \\
\hline Krt 19 & CACTACAGCCACTACTACACGA & CTCATGCGCAGAGCCTGTT \\
\hline Wntl & CATCTTCGCAATCACCTCCG & GTGGCATTTGCACTCTTGG \\
\hline Cyclin DI & TAGGCCCTCAGCCTCACTC & CCACCCCTGGGATAAAGCA \\
\hline Gapdh & АСТСССАСТСТТССАССТТС & TCTTGCTCAGTGTCCTTGC \\
\hline
\end{tabular}

\section{Western Blot Analysis}

After the indicated treatment, cells were washed 3 times with PBS and lysed with cell lysis buffer supplemented with protease inhibitor cocktail. Nuclear fractions were prepared using a commercial Nuclear/Cytosol Fractionation Kit (Thermo Fisher Scientific, USA). A total of $20 \mu \mathrm{g}$ isolated protein was used for $10 \%$ sodium dodecyl sulfate-polyacrylamide gel electrophoresis (SDS-PAGE) and transferred to polyvinylidene fluoride (PVDF) membranes. After washing 3 times with Tris Buffered Saline Tween (TBST), the membranes were blocked with $5 \%$ non-fat milk for $1 \mathrm{~h}$ at room temperature (RT). Then, the membranes were sequentially incubated with primary antibody overnight in a cold room. The following primary antibodies were used: Gpr40 (1:1000, Santa Cruz, sc-28416) Wnt-1 (1:2000, Santa Cruz, sc-514531) Cyclin D1 (1:2000, Santa Cruz, sc8396) $\beta$-actin (1:5000, Santa Cruz, sc-8432). After the reaction, the membrane was incubated with corresponding horseradish peroxidase (HRP)-conjugated secondary antibody for $2 \mathrm{~h}$ at room temperature. Enhanced chemiluminescence was used to visualize the immunoreactive bands (Thermo Fisher Scientific, USA). The following antibodies were used Gpr40 (1:1000, ab236285, Abcam); Wnt-1 (1:2000, sc-514531, Santa Cruz); Cyclin D1 (1:2000, sc-8396, Santa Cruz Biotechnology); $\beta$-Catenin (\#9582, Cell Signaling Technology, USA); Lamin B1 (\#12586, Cell Signaling Technology, USA); $\beta$-actin (1:5000, Santa Cruz Biotechnology, sc-8432), Anti-rabbit IgG, HRP-linked antibody (\#7074, Cell Signaling Technology, USA); Anti-mouse IgG, HRP-linked antibody (\#7076, Cell signaling Technology, USA).

\section{Enzyme-Linked Immunosorbent Assay (ELISA)}

The protein secretion of target genes into the culture medium was determined using ELISA kits (R\&D Systems, USA) in accordance with the manufacturer's instructions. In brief, the cell culture media was collected and centrifuged to remove cell pellets. The protein concentration in the supernatant was measured by the bicinchoninic acid (BCA) protein assay method with a commercial kit (Thermo Fisher Scientific, 23227). The resulting data was recorded using 96-plate reader spectrometry. The cytokine concentration was calculated and normalized to the protein concentration of the sample.

\section{2',7'-Dichlorodihydrofluorescein Diacetate (DCFH-DA) Staining}

After the indicated treatment, the production of ROS was measured by staining ESCs with the fluorescent dye DCFH-DA (Thermo Fisher Scientific, USA). Briefly, the cells were washed 3 times and loaded with $5 \mu \mathrm{M}$ DCFHDA, then incubated for $30 \mathrm{~min}$ at a temperature of $37^{\circ} \mathrm{C}$ in darkness. A confocal microscope (Zeiss, Germany) was used to quantify the resulting DCFH-DA fluorescence under $488 \mathrm{~nm}$ excitation light.

\section{Lactate Dehydrogenase (LDH) Release}

After necessary treatment, the release of LDH from ESCs was assayed using a commercial kit (Thermo Fisher Scientific, USA). Cell culture supernatant $(50 \mu \mathrm{L})$ was collected and mixed with equal volume reaction agent and incubated at $37^{\circ} \mathrm{C}$ for $30 \mathrm{~min}$. After that, $50 \mu \mathrm{L}$ stop buffer was added to stop the reaction. The optical density (OD) value at $490 \mathrm{~nm}$ was recorded to index LDH release. 
A

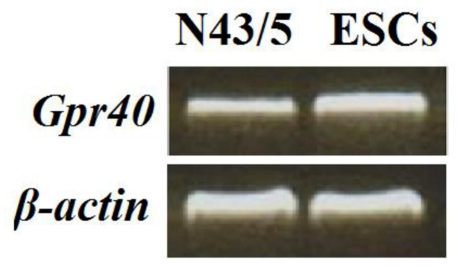

B

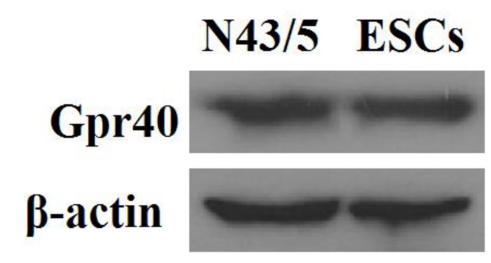

C

Gpr40

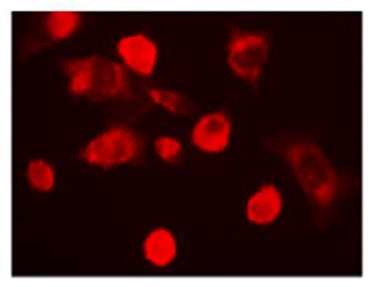

D

Gpr40

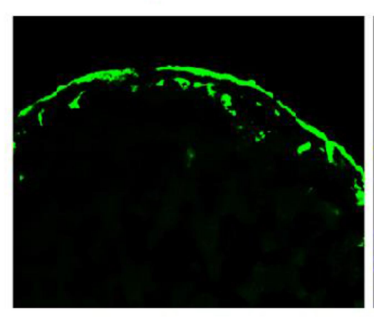

DAPI

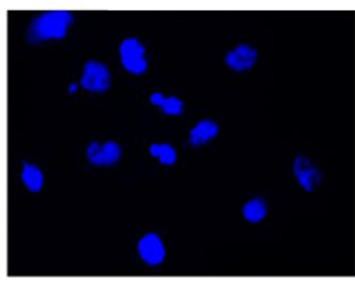

DAPI

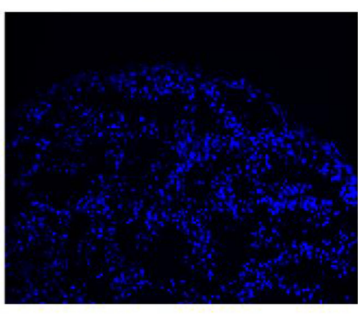

\section{Merged}

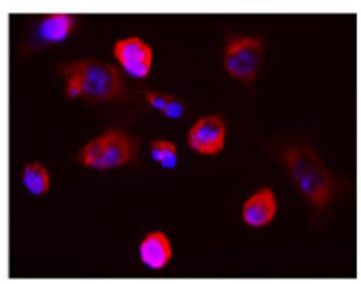

\section{Merged}

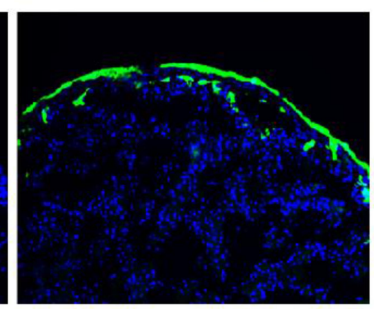

Figure I Gpr40 is expressed in mouse epidermal stem cells (ESCs). (A) Expression of Gpr40 at the gene level in mouse ESCs was measured by reverse transcription PCR with murine N43/5 cell line was used as a positive control. (B) Expression of Gpr40 at the protein level in mouse ESCs was measured by Western blot analysis. (C) Immunocytochemical staining of Gpr40 in ESCs. (D) Immunocytochemical staining of Gpr40 in skin tissue.

\section{Immunofluorescence}

Skin biopsies were taken from the backs of mice. Mouse skin sections were routinely fixed with formalin and embedded in paraffin. After demasking with Protease XXIV (Sigma-Aldrich, USA) in $20 \mathrm{mM}$ Tris $\mathrm{pH} 7.9$, the skin sections were incubated with the primary antibody against Gpr40 (1:1000, Santa Cruz, sc-28416), followed by incubation with donkey anti-rabbit IgG Alexa 488 (Invitrogen, USA). Sections were mounted with 4',6-diamidino-2-phenylindole (DAPI).

For cell staining, $10 \%$ formalin to was added to the dish and fixed at room temperature for $10 \mathrm{~min}$. Cells were then permeabilized with $0.1 \%$ Triton $\times 100$ for $10 \mathrm{~min}$. After blocking with $10 \%$ normal goat serum for $1 \mathrm{~h}$ at room temperature, were incubated with the primary antibody against Gpr40 (1:1000, Santa Cruz, sc-28416) overnight at $4^{\circ} \mathrm{C}$, followed by incubation with donkey anti- rabbit IgG Alexa 594 (Invitrogen, USA). Sections were mounted with 4',6-diamidino-2-phenylindole (DAPI). Fluorescent signals were visualized with a fluorescent microscope.

\section{Statistical Analysis}

All experiments were repeated three times. The data from all experiments were analyzed using SPSS software (Version 19). The results are expressed as the means \pm standard deviation (S.D.). Statistical analysis was determined based on analysis of variance (ANOVA), followed by Bonferroni's post hoc test. Statistical significance is represented by a value of $\mathrm{p}<0.05$.

\section{Results}

First, we investigated whether Gpr40 is expressed in ESCs. As shown in Figure 1A and B, we established 
results demonstrating the presence of Gpr40 in ESCs with N43/5 cells as a positive control. ${ }^{21}$ Expression of Gpr40 was confirmed by immunocytochemical staining in both ESCs (Figure 1C) and skin tissue samples taken from the backs of mice (Figure 1D). We further found that UB-V radiation significantly suppressed Gpr40 expression, as shown in Figure 2A and B. Next, we studied the effect of the Gpr40 agonist GW9508 on UV-B-induced upregulation of oxidative stress. The results in Figure 3 of our experimentation exhibit the ability of UV-B to induce an increase in ROS generation of 3.5-fold, which was dosedependently reduced to 1.5 -fold by Gpr40 agonism. We moved on to examine the effect of Gpr40 agonism on cell death as measured by the release of LDH from ESCs. Indeed, agonism of Gpr40 dose-dependently reduced the initial UV-B-induced increase in LDH release of roughly 5 -fold to only roughly 2 -fold baseline, as demonstrated in Figure 4.

Inflammation is a critical factor in the development of most diseases, including the effects of UV-B radiation. We examined the expression of the proinflammatory cytokines IL-1 $\beta$ and IL-8 in UV-B-irradiated cells. Our results indicate that Gpr40 agonism can ameliorate the radiationinduced increase in these cytokines at both the mRNA and protein levels, as shown in Figure 5. The increases in the mRNA expression of IL-1 $\beta$ and IL- 8 of 3.8- and 4.5 -fold were mitigated to only roughly 1.75 -fold by Gpr40 agonism (Figure 5A). Similar results were observed at the protein level (Figure 5B). We determined the effects of GW9508 on UV-B-induced impairment of the capacities of ESCs by measuring the levels of integrin $\beta 1$ and Krt19. Both of these protective factors were significantly downregulated by exposure to UV-B, while agonism of Gpr40 by GW9508 rescued integrin $\beta 1$ and Krt19 expression to near baseline, as shown in Figure 6. To further clarify whether the effects of GW9508 on the capacities of ESCs against UV-B are associated with the suppression of ROS, the antioxidant N-acetyl-L-cysteine (NAC) was used. The results in Supplementary Figure 1 indicate that UV-B-induced downregulation of integrin $\beta 1$ and Krt19 were restored by NAC in a dose-dependent manner. These results suggest that the protective effects of GW9508 on the capacities of ESCs might be associated with the suppression of ROS.

The Wnt1 and $\beta$-catenin proteins play a key role in maintaining the capacities of ESCs and have been

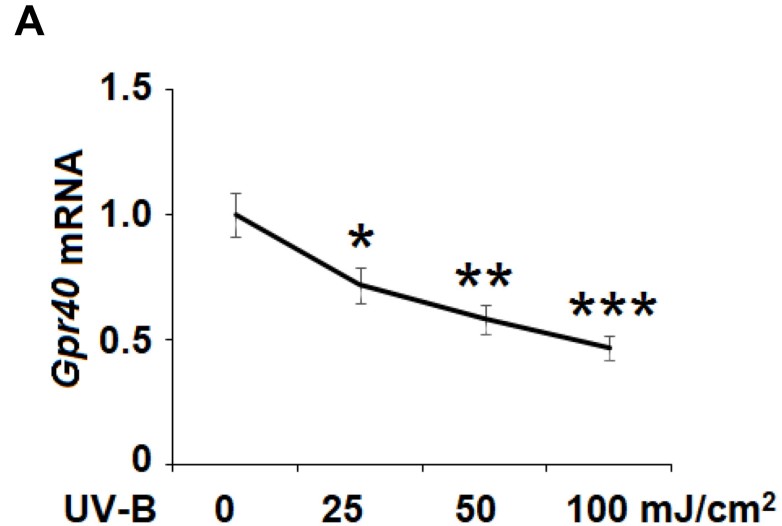

B

$\begin{array}{lllll}\text { UV-B } & 0 & 25 & 50 & 100 \mathrm{~mJ} / \mathrm{cm}^{2}\end{array}$
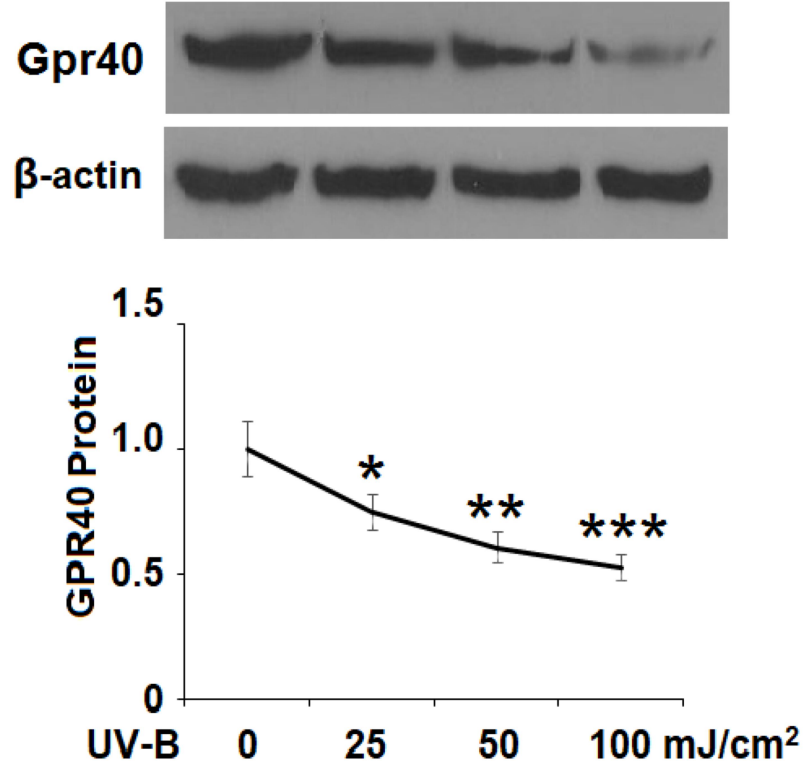

Figure 2 Ultraviolet-B (UV-B) reduced the expression of Gpr40 in epidermal stem cells (ESCs). Cells were exposed to ultraviolet-B (UV-B) $\left(25,50,100 \mathrm{~mJ} / \mathrm{cm}^{2}\right)$ for 24 h. (A) mRNA level of Gpr40. (B) Protein level of Gpr40 (*, **, ***, P<0.05, 0.0I, 0.001 vs previous column group, $n=4$ ).

identified as mediators of resistance to UV-B radiationinduced damage. As shown in Figure 7A and B, there was a significant UV-B-induced decrease in the expression of Wnt1 and cyclin D1 at both the mRNA and protein levels. Remarkably, agonism of Gpr40 by GW9508 rescued nuclear $\beta$-catenin expression to near baseline (Figure 7C). Finally, we determined whether the effects of GW9508 against UV-B-induced cellular damage are dependent on Gpr40 signaling by performing a Gpr40 


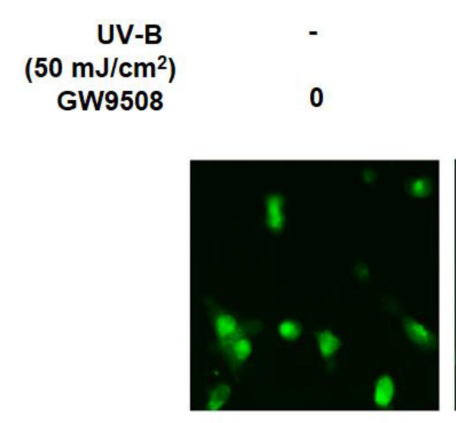
$+$
0
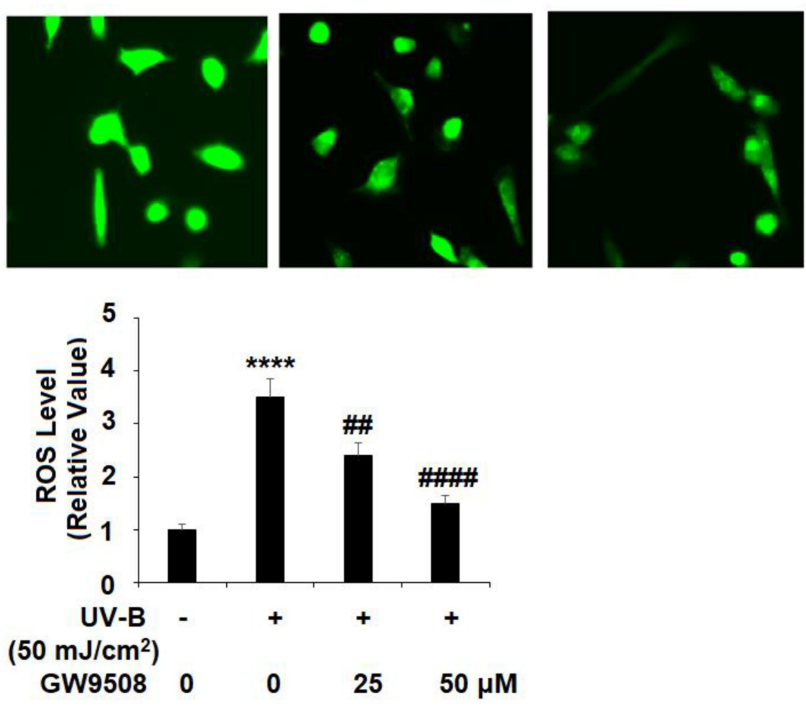

Figure 3 The Gpr40 agonist GW9508 protected against ultraviolet-B (UV-B)-induced oxidative stress in epidermal stem cells (ESCs). Cells were exposed to UV-B (50 mJ/ $\left.\mathrm{cm}^{2}\right)$ with or without GW9508 $(25,50 \mu \mathrm{M})$ for $24 \mathrm{~h}$. Generation of reactive oxygen species (ROS) was measured by DCFH-DA staining $(* * * *$, P<0.000I vs vehicle control; \#, \#\#, $\mathrm{P}<0.01$, 0.000 I vs UV-B group, $\mathrm{n}=4$ ).

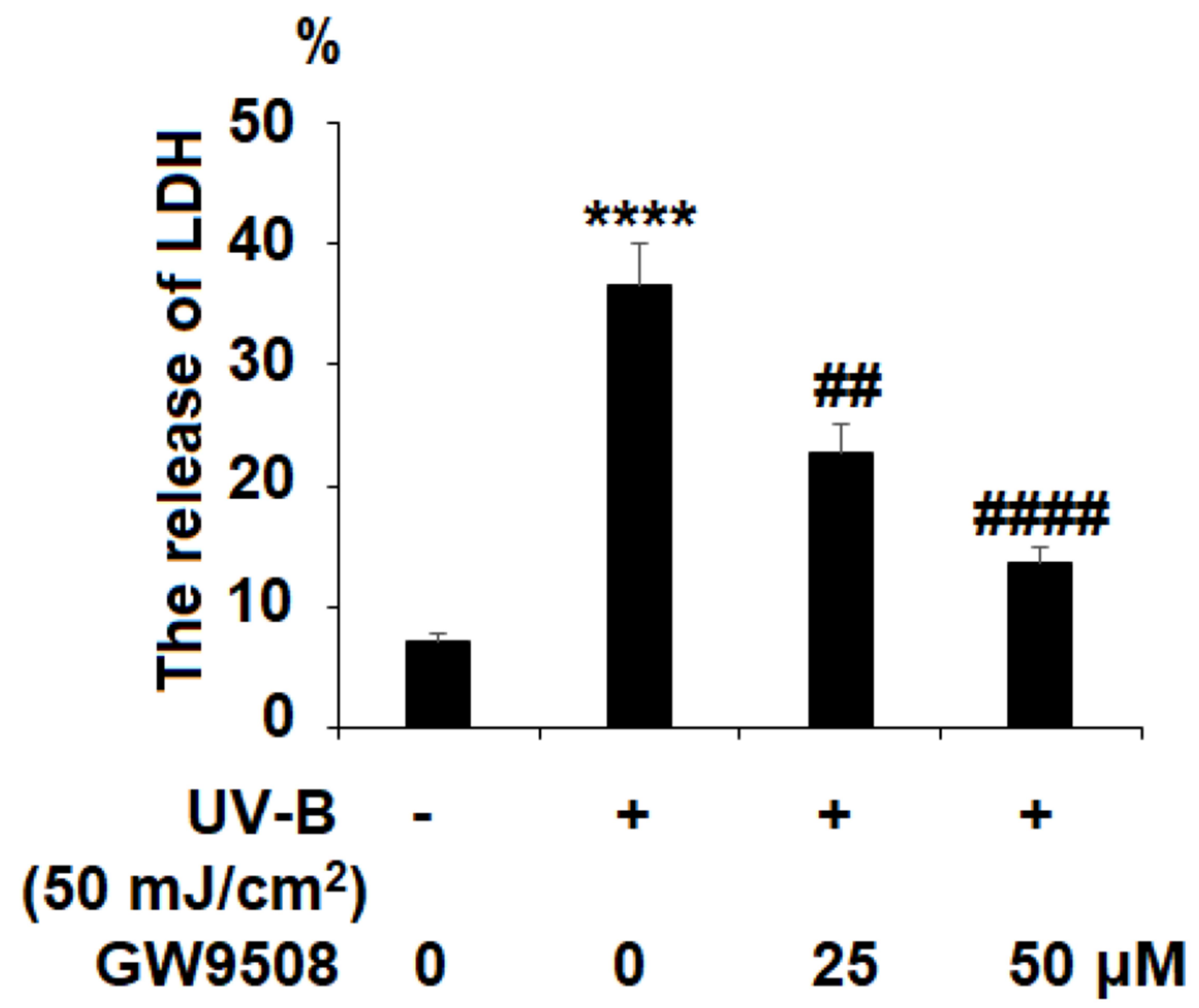

Figure 4 GW9508 reduced ultraviolet-B (UV-B)-induced release of lactate dehydrogenase (LDH) in epidermal stem cells (ESCs). Cells were exposed to UV-B (50 mJ/cm²) with or without GW9508 $(25,50 \mu \mathrm{M})$ for $24 \mathrm{~h}$. The release of $\mathrm{LDH}$ was measured $(* * * *, \mathrm{P}<0.000 \mathrm{I}$ vs vehicle control; \#\#, \#\#, P<0.0I, $0.000 \mathrm{I}$ vs $U$ V-B group, $\mathrm{n}=4)$. 
A
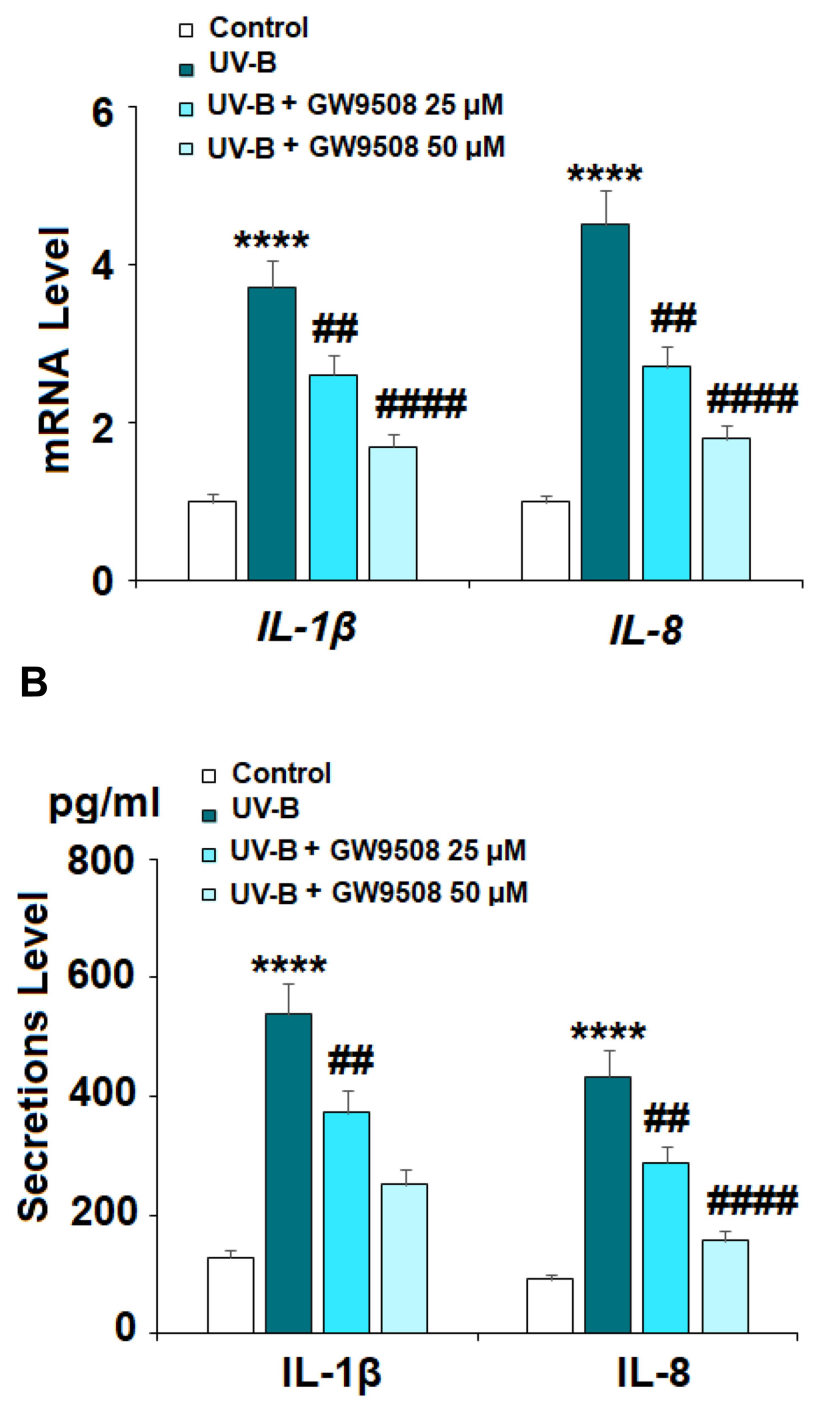

Figure 5 GW9508 suppressed ultraviolet-B (UV-B)-induced expression and secretion of pro-inflammatory cytokines. Cells were exposed to UV-B $\left(50 \mathrm{~m} / / \mathrm{cm}^{2}\right)$ with or without GW9508 $(25,50 \mu \mathrm{M})$ for $24 \mathrm{~h}$. (A) mRNA of IL-I $\beta$ and IL-8. (B) Secretions of IL-I $\beta$ and IL-8 (****, P<0.000I vs vehicle control; \#\#, \#\#\#, $\mathrm{P}<0.01,0.0001$ vs UV-B group, $n=4$ ).

antagonism experiment using the specific Gpr40 antagonist GW1100. Indeed, antagonism of Gpr40 completely abolished the ability of GW9508 to rescue the expression of integrin $\beta 1$ and Krt19 (Figure 8), suggesting the involvement of Gpr40 in this process.

\section{Discussion}

There is an ever-present risk of skin damage from UV$B$ due to its natural emission from the sun. Although most UV-B is absorbed in the ozone, some radiation manages to penetrate the atmosphere and come into contact with human skin. Especially over long periods, UV-B exposure can cause significant damage. In the present study, we aimed to test the effects of Gpr40 agonism by GW9508 in UV-B-induced damage to ESCs, the pluripotent stem cells of the epidermis. Importantly, we identified Gpr40 expression in murine ESCs for what we believe to be the first time. We further established that agonism of Gpr40 by GW9508 could mitigate UV-B-induced damage in this cell type by reducing oxidative stress, inflammation, and rescuing the expression of protective factors, including integrin $\beta 1$ and $\mathrm{Krt1}$, while preserving cell viability. Furthermore, we demonstrate that these effects are mediated through the Wnt $1 / \beta$-catenin pathway. Importantly, the beneficial effects of Gpr40 were verified by using another agonist TAK-875. Treatment with TAK-875 exerts a robust protective action against UV-B exposure by increasing integrin $\beta 1$ and Krt1 (Data not shown).

Oxidative stress, often manifested through the overproduction of ROS, is a major aspect of many diseases, including UV-B-induced skin damage. Recent studies utilizing murine models have demonstrated the role of ROS in UV-B-induced insult in a variety of diseases ranging from skin cancer to cataracts. ${ }^{22,23}$ Indeed, UV$\mathrm{B}$ overexposure even induces oxidative stress in flora, demonstrating the over-arching risk of ROS overproduction due to UV-B. ${ }^{24}$ This prevalent oxidative stressinducing ability of UV-B is, therefore, a key point of focus in the search for therapies to mitigate UV-Binduced injury. The results of the present study demonstrate a clear ability of Gpr40 agonism to limit UV-Binduced ROS production. Both GW9508 and TAK-875 effectively agonize Gpr40 but they also activate other $\mathrm{G}$ protein receptors. Kim et al showed that high doses of TAK-875 $(50,100,250$ and $500 \mu \mathrm{M})$ dose-responsively induce the death of HepG2 cells, ${ }^{25}$ which was found to be associated with the induction of hepatic ROS generation. Philippe et al report that a high dose of GW9508 $(100 \mu \mathrm{M})$ decreases the proliferation of osteoblast precursor cell line RAW264.7. Mechanistically, the study suggests that high-dose treatment with GW9508 induced mitochondrial ROS production and bone cell death, which is associated 


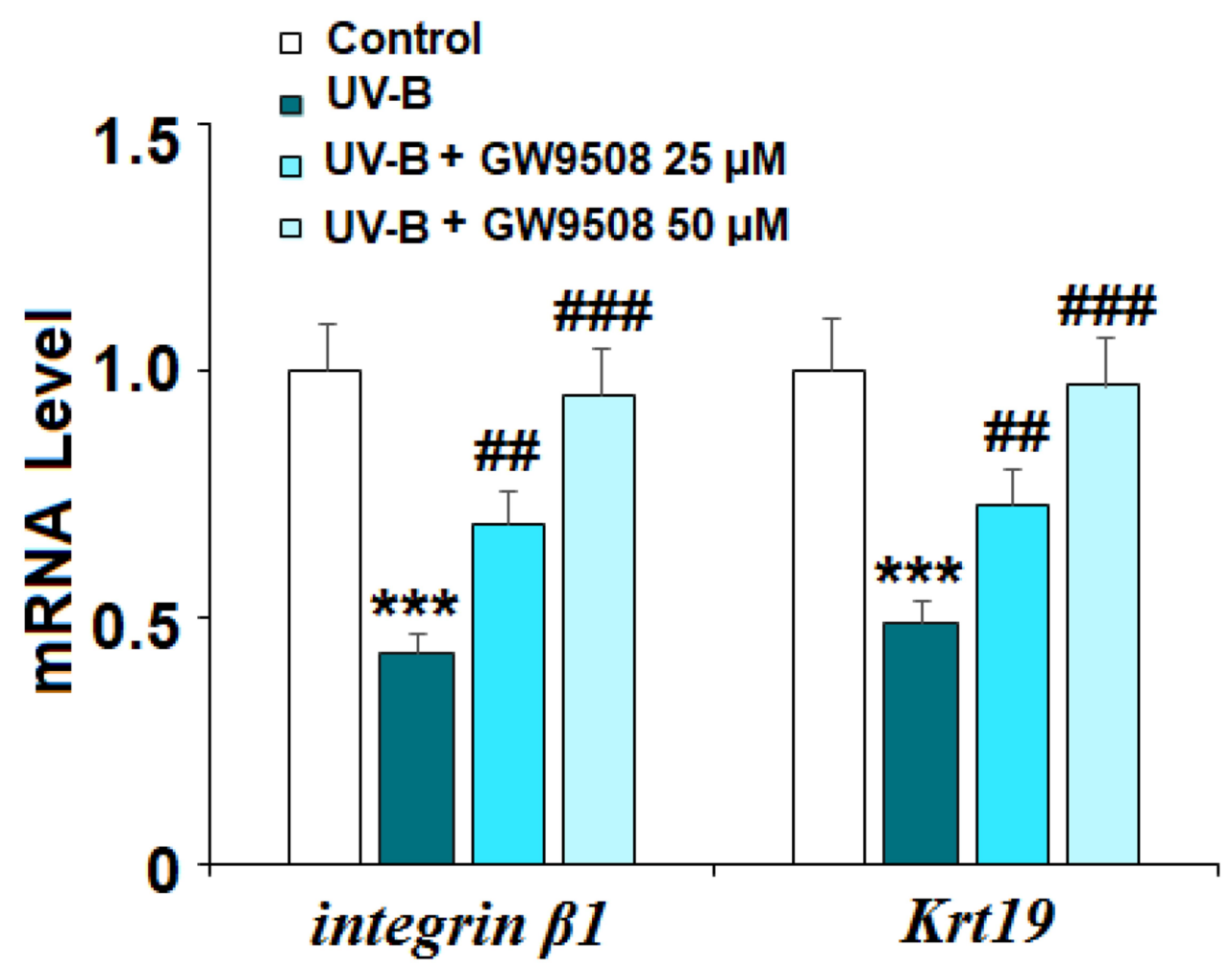

Figure 6 GW9508 prevented ultraviolet-B (UV-B) exposure-induced impairment in the capacities of ESCs. Cells were exposed to UV-B (50 mJ/cm²) with or without GW9508 $(25,50 \mu \mathrm{M})$ for 24 h. mRNA of integrin $\beta$ I and Krt 19 was measured by real-time PCR analysis (***, P<0.00I vs vehicle control; \#, \#\#, P<0.0I, 0.00 I vs UV-B group, $n=4$ ).

with necrosis mechanisms. ${ }^{26}$ In both of these studies, high doses of Gpr40 agonists were used. In our study, we used 25 or $50 \mu \mathrm{M}$ GW9508 to activate Gpr40 in ESCs. A possible explanation is that the low doses of Gpr40 agonists could have a Gpr40-dependent mechanism while high doses of agonists could have a Gpr40independent role. Another reason is that different types of cells might respond differently to GW9508 and TAK-875. Further investigations will help us to uncover the complete underlying mechanism.

Acute and chronic inflammation is another key aspect of an array of diseases, including melanoma, which can be a life-threatening outcome of UV-Binduced cellular damage. ${ }^{27}$ Dysregulation of the expression of proinflammatory cytokines is the most common cause of pathological inflammation. The role of proinflammatory cytokines in UV-B-induced cellular damage has long been recognized. ${ }^{28}$ Recent research suggests that the high melanin production in dark-skinned individuals developed as a natural mechanism to protect against UV-B-induced epidermal inflammation. ${ }^{29}$ Here, we found that agonism of Gpr40 could reduce the UVB-induced increase in the expression of IL- $1 \beta$ and IL- 8 in ESCs.

Krt19 and integrin $\beta 1$ serve vital protective roles in maintaining skin homeostasis, the functions of ESCs, and preventing an excessive inflammatory response. The expression of Krt19 has been shown to be reduced by exposure to UV-B, and recent research suggests that rescuing Krt19 expression can preserve the physiological functions of ESCs. ${ }^{30}$ The integrin family has long been associated with mediating the effects of UV radiation. An integrin $\beta 1$-related $\alpha 6$-integrin has been shown to be downregulated in skin cells exposed to UV-B. ${ }^{31}$ Another integrin, integrin $\alpha 3$, has been used as a marker for ESC functionality. ${ }^{32}$ In our experiments, we found that agonism of Gpr40 by GW9508 rescues the reduction in Krt19 and integrin $\beta 1$ induced by UV-B 
A
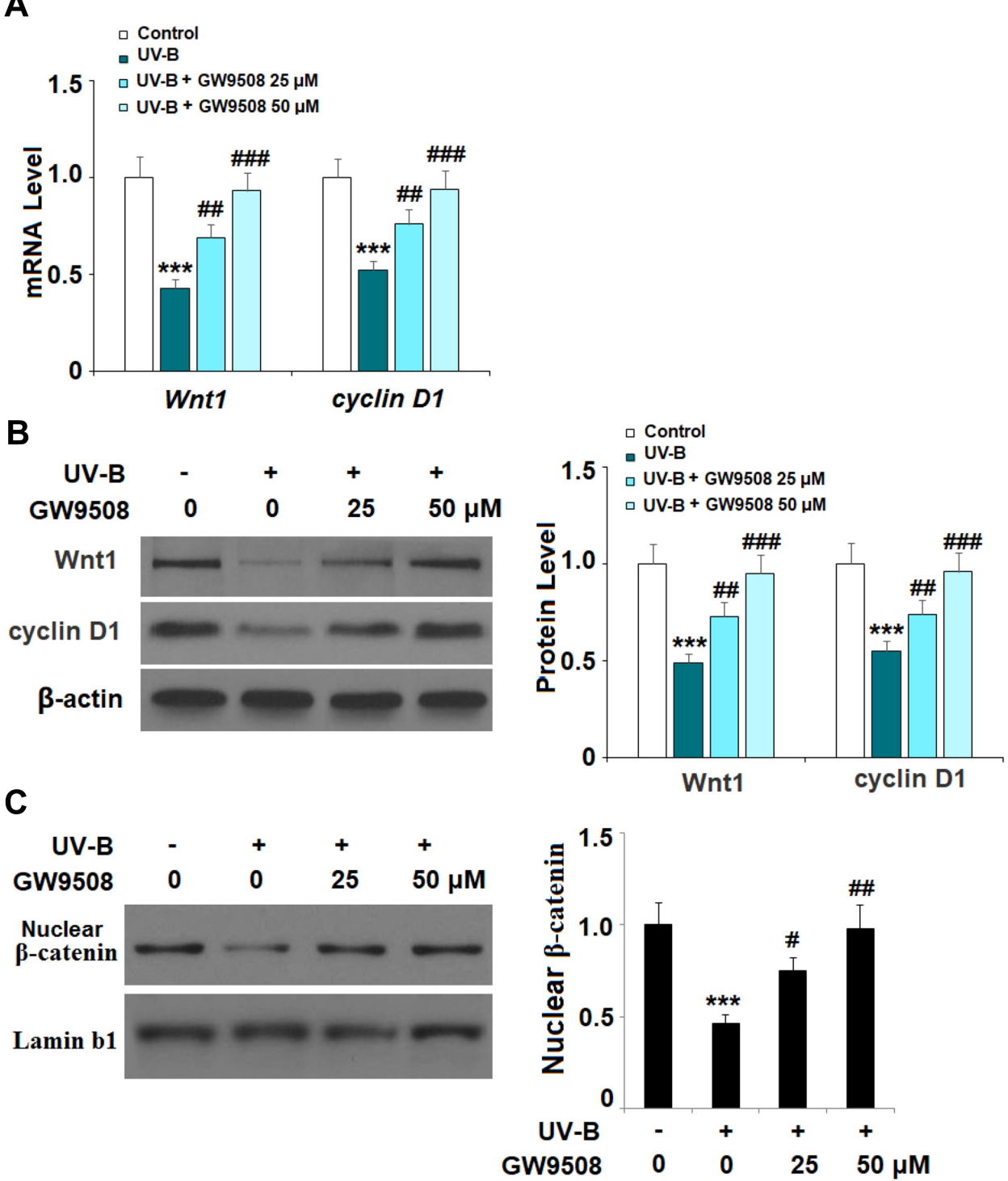

Figure 7 GW9508 inhibited ultraviolet-B (UV-B)-induced decrease of Wnt/ $/$-catenin pathway proteins. Cells were exposed to UV-B (50 mJ/cm²) with or without GW9508 $(25,50 \mu \mathrm{M})$ for 24 h. (A) Real-time PCR analysis of Wnt I and cyclin DI at the mRNA level. (B) Western blot analysis of Wnt I and cyclin DI at the protein level. (C) Nuclear levels of $\beta$-catenin were measured (***, $P<0.00$ I vs vehicle control; \#, \#, \#\#, $P<0.05,0.01,0.00$ I vs UV-B group, $n=4$ ).

radiation. As Krt19 and integrin $\beta 1$ serve important protective roles against UV-B-induced cellular damage, agonism of Gpr40 may play a key role in maintaining the functionality of ESCs during insult from UV-B. Activation of $\mathrm{Wnt} /$ integrin $\beta 1$ signaling results in increased levels of Wnt1 and cyclin D1, which play important roles in maintaining the protection of ESCs. $^{33} \mathrm{~A}$ recent study investigating the involvement of GPR30, a $\mathrm{G}$ protein-coupled receptor for estrogen, in mediating the effects of UV radiation in ESCs found that GPR30 agonism could protect against UV-Binduced damage by increasing the protein expression of Wnt1 and cyclin D1. ${ }^{34}$ Here, we found that agonism of Gpr40 restores the expression of these protective factors. Thus, the canonical $\mathrm{Wnt} / \beta$-catenin plays a role in mediating the protective effects of the Gpr40 agonism against UV-B radiation in ESCs. This study lays the groundwork for further study, which is required to broaden our understanding of the role of Gpr40 in UVB-induced skin damage and the underlying mechanism. 


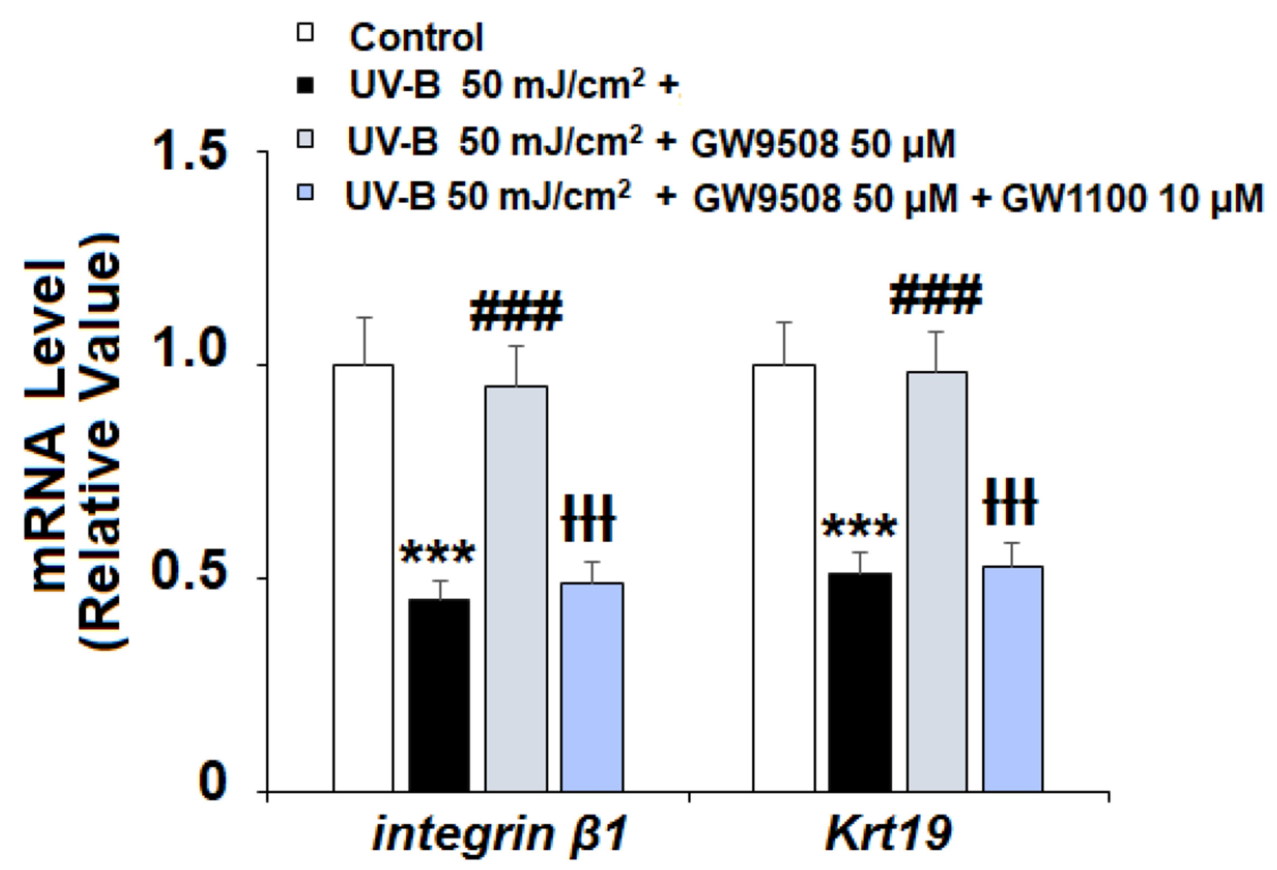

Figure 8 The effects of GW9508 in maintaining the capacities of ESCs are dependent on Gpr40. Cells were exposed to UV-B (50 mJ/cm²) in the presence or absence of GW9508 $(50 \mu \mathrm{M})$ or the Gpr40 antagonist GWII00 (I0 $\mu \mathrm{M})$. mRNA of integrin $\beta I$ and Krt 19 was detected with real-time PCR (***, P<0.00I vs vehicle control; \#\#, $P<0.00$ I vs UV-B group; HI, $P<0.00$ I vs $U V-B+G W 9508$ group, $n=4)$.

\section{Disclosure}

The authors report no conflicts of interest for this work. Chengkuan Sun and Yulin Li are co-first authors.

\section{References}

1. Lee SE, Lee SH. Skin Barrier and Calcium. Ann Dermatol. 2018;30 (3):265-275. doi:10.5021/ad.2018.30.3.265

2. Zhu J, Wang P, Yu Z, et al. Advanced glycosylation end product promotes forkhead box $\mathrm{O} 1$ and inhibits Wnt pathway to suppress capacities of epidermal stem cells. Am J Transl Res. 2016;8 (12):5569.

3. Grant WB. Ecologic Studies of Solar UV-B Radiation and Cancer Mortality Rates. InVitamin D Analogs in Cancer Prevention and Therapy. Springer; 2003:371-377.

4. Viiri J, Jauhonen HM, Kauppinen A, et al. Cis-urocanic acid suppresses UV-B-induced interleukin-6 and-8 secretion and cytotoxicity in human corneal and conjunctival epithelial cells in vitro. Mol Vis. 2009; $15: 1799$.

5. Narbutt J, Lesiak A, Sysa-Jedrzejowska A, et al. Repeated low dose ultraviolet (UV) B exposures of humans induce limited photoprotection against the immune effects of erythemal UVB radiation. Br J Dermatol. 2007;156(3):539-547. doi:10.1111/j.1365-2133.2006.07670.x

6. Goyal S, Amar SK, Srivastav AK, et al. ROS mediated crosstalk between endoplasmic reticulum and mitochondria by Phloxine $\mathrm{B}$ under environmental UV irradiation. $J$ Photochem Photobiol B. 2016;161:284-294. doi:10.1016/j.jphotobiol.2016.05.031

7. Huelsken J. The Wnt signalling pathway. J Cell Sci. 2002;115 (21):3977-3978. doi:10.1242/jcs.00089

8. Zhao XK, Cheng Y, Cheng ML, et al. Focal adhesion kinase regulates fibroblast migration via integrin beta- 1 and plays a central role in fibrosis. Sci Rep. 2016;6(1):19276. doi:10.1038/srep19276
9. Ko H, Hasegawa H, Ochiai T, et al. Loss of Basal Cell Character in Regenerating Oral Squamous Epithelium with Altered Expression of Desmoglein 1, Desmocollin 3 and Keratin 19. J Hard Tissue Biol. 2019;28(1):43-50. doi:10.2485/jhtb.28.43

10. Woodward WA, Chen MS, Behbod F, Alfaro MP, Buchholz TA, Rosen $\mathrm{JM}$. Wnt $/ \beta$-catenin mediates radiation resistance of mouse mammary progenitor cells. Proc National Acad Sci. 2007;104(2):618-623.

11. Chang Y, Yu J. The protective effects of TGR5 against ultraviolet B irradiation in epidermal stem cells. J Cell Biochem. 2019;120 (9):15038-15044. doi:10.1002/jcb.28765

12. Rayasam GV, Tulasi VK, Davis JA, Bansal VS. Fatty acid receptors as new therapeutic targets for diabetes. Expert Opin Ther Targets. 2007;11(5):661-671. doi:10.1517/14728222.11.5.661

13. Edfalk S, Steneberg P, Edlund H. Gpr40 is expressed in enteroendocrine cells and mediates free fatty acid stimulation of incretin secretion. Diabetes. 2008;57(9):2280-2287. doi:10.2337/db080307

14. Ueno H, Ito R, Abe SI, et al. Gpr40 full agonism exerts feeding suppression and weight loss through afferent vagal nerve. PLoS One. 2019;14(9):9. doi:10.1371/journal.pone.0222653

15. Gagnon L, Leduc M, Thibodeau JF, Leblond FA, Laurin P, Grouix B. FO008 FATTY ACID RECEPTORS Gpr40/GPR84: TWO PROMISING TARGETS IN KIDNEY FIBROSIS. Nephrology Dialysis Transplantat. 2019;34(Supplement_1):gfz096-FO008. doi:10.1093/ndt/gfz096.FO008

16. Li MH, Chen W, Wang LL, et al. RLA8-A New and Highly Effective Quadruple PPAR- $\alpha / \gamma / \delta$ and Gpr40 Agonist to Reverse Nonalcoholic Steatohepatitis and Fibrosis. J Pharmacol Exp Therapeutics. 2019;369(1):67-77. doi:10.1124/jpet.118.255 216

17. Chen JJ, Gong YH, He L. Role of Gpr40 in pathogenesis and treatment of Alzheimer's disease and type 2 diabetic dementia. $J$ Drug Target. 2019;27(4):347-354. doi:10.1080/1061186X.2018.149 1979 
18. Suski M, Kiepura A, Wiśniewska A, et al. Anti-atherosclerotic action of GW9508-Free fatty acid receptors activator-In apoE-knockout mice. Pharmacol Rep. 2019;71(4):551-555. doi:10.1016/j.pharep.2019.02.014

19. Hashimoto T, Mogami H, Tsuriya D, et al. G-protein-coupled receptor 40 agonist GW9508 potentiates glucose-stimulated insulin secretion through activation of protein kinase $\mathrm{C} \alpha$ and $\varepsilon$ in INS-1 cells. PLoS One. 2019;14:9.

20. Yang RH, Qi SH, Shu B, et al. Epidermal stem cells (ESCs) accelerate diabetic wound healing via the Notch signalling pathway.. Biosci Rep. 2016;36(4):pii: e00364. doi:10.1042/BSR20160034

21. Hernández-Cáceres MP, Toledo-Valenzuela L, Díaz-Castro F, et al. Palmitic Acid Reduces the Autophagic Flux and Insulin Sensitivity Through the Activation of the Free Fatty Acid Receptor 1 (FFAR1) in the Hypothalamic Neuronal Cell Line N43/5. Front Endocrinol (Lausanne). 2019;10:176. doi:10.3389/fendo.2019.00176

22. Hua H, Yang T, Huang L, et al. Protective effects of lanosterol synthase up-regulation in UV-B induced oxidative stress. Front Pharmacol. 2019;10:947. doi:10.3389/fphar.2019.00947

23. Li K, Zhang M, Chen H, et al. Anthocyanins from black peanut skin protect against UV-B induced keratinocyte cell and skin oxidative damage through activating Nrf 2 signaling. Food Funct. 2019;10 (10):6815-6828. doi:10.1039/C9FO00706G

24. Cassia R, Amenta M, Fernández MB, Nocioni M, Dávila V. The Role of Nitric Oxide in the Antioxidant Defense of Plants Exposed to UVB Radiation. Reactive Oxygen, Nitrogen and Sulfur Species in Plants: production, Metabolism. Signaling Defense Mechanisms. 2019;555-572.

25. Kim M, Gu GJ, Koh YS, et al. Fasiglifam (TAK-875), a G ProteinCoupled Receptor 40 (Gpr40) Agonist, May Induce Hepatotoxicity through Reactive Oxygen Species Generation in a Gpr40-Dependent Manner. Biomol Ther (Seoul). 2018;26(6):599-607. doi:10.4062/ biomolther.2017.225

26. Philippe C, Wauquier F, Léotoing L, Coxam V, Wittrant Y. GW9508, a free fatty acid receptor agonist, specifically induces cell death in bone resorbing precursor cells through increased oxidative stress from mitochondrial origin. Exp Cell Res. 2013;319(19):3035-3041. doi:10.1016/j.yexcr.2013.08.013
27. Satou G, Maji D, Isamoto T, Oike Y, Endo M. UV-B-activated B16 melanoma cells or HaCaT keratinocytes accelerate signaling pathways associated with melanogenesis via ANGPTL 2 induction, an activity antagonized by Chrysanthemum extract. Exp Dermatol. 2019;28(2):152-160

28. Bae JY, Choi JS, Kang SW, Lee YJ, Park J, Kang YH. Dietary compound ellagic acid alleviates skin wrinkle and inflammation induced by UV-B irradiation. Exp Dermatol. 2010;19(8):e182-e190. doi:10.1111/j.1600-0625.2009.01044.x

29. Lin TK, Man MQ, Abuabara K, et al. By protecting against cutaneous inflammation, epidermal pigmentation provided an additional advantage for ancestral humans. Evol Appl. 2019;12(10):1960-1970. doi:10.1111/eva.12858

30. Wang X, Liu X, Zhao Y, Sun H, Wang Y. Cytoprotective role of S14G-humanin (HNG) in ultraviolet-B induced epidermal stem cells injury. Biomed Pharmacother. 2019;110:248-253. doi:10.1016/j. biopha.2018.11.059

31. Krengel S, Stark I, Geuchen C, et al. Selective down-regulation of the $\alpha 6$-integrin subunit in melanocytes by UVB light. Exp Dermatol. 2005;14(6):411-419. doi:10.1111/j.0906-6705.2005.00295.x

32. Tomellini E, Fares I, Lehnertz B, et al. Integrin- $\alpha 3$ Is a Functional Marker of Ex Vivo Expanded Human Long-Term Hematopoietic Stem Cells. Hum Pathol. 2012;43(7):1044-1050.

33. Zhang J, Gill AJ, Issacs JD, et al. The Wnt/ $\beta$-catenin pathway drives increased cyclin D1 levels in lymph node metastasis in papillary thyroid cancer. Hum Pathol. 2012;43(7):1044-1050. doi:10.1016/j. humpath.2011.08.013

34. Zhang Y, Li L, Xu Y, Zhao X, Li F. Protective mechanism of GPR30 agonist G1 against ultraviolet B-induced injury in epidermal stem cells. Artificial Cells Nanomed Biotech. 2019;47(1):4165-4171. doi:10.1080/21691401.2019.1687497
Drug Design, Development and Therapy

\section{Publish your work in this journal}

Drug Design, Development and Therapy is an international, peerreviewed open-access journal that spans the spectrum of drug design and development through to clinical applications. Clinical outcomes, patient safety, and programs for the development and effective, safe, and sustained use of medicines are a feature of the journal, which has also

\section{Dovepress}

been accepted for indexing on PubMed Central. The manuscript management system is completely online and includes a very quick and fair peer-review system, which is all easy to use. Visit http://www. dovepress.com/testimonials.php to read real quotes from published authors. 